\title{
Analytical and Finite Element Modeling of the Dynamic Characteristics of a Linear Feeding Stage with Different Arrangements of Rolling Guides
}

\author{
Jyh-Cheng Chang ${ }^{1}$ and Jui-Pin Hung ${ }^{2}$ \\ ${ }^{1}$ Department of Automation Engineering, Nan Kai University of Technology, No. 568 Zhongzheng Road, Caotun Township, \\ Nantou County 54243, Taiwan \\ ${ }^{2}$ Graduate Institute of Precision Manufacturing, National Chin-Yi University of Technology, No. 57, Section 2, Zhongshan Road, \\ Taiping District, Taichung 41170, Taiwan
}

Correspondence should be addressed to Jui-Pin Hung; hungjp@ncut.edu.tw

Received 22 February 2014; Accepted 6 April 2014; Published 1 July 2014

Academic Editor: Her-Terng Yau

Copyright (C) 2014 J.-C. Chang and J.-P. Hung. This is an open access article distributed under the Creative Commons Attribution License, which permits unrestricted use, distribution, and reproduction in any medium, provided the original work is properly cited.

\begin{abstract}
This study was aimed at investigating the dynamic behaviors of the linear driven feeding stage by means of the analytical and finite element modeling approaches. To assess the dynamic characteristics of the stages with different linear guide arrangements, the finite element model of the stages was created, in which the linear components with rolling interface were accurately modeled based on the Hertzian theory. On the other hand, the analytically mathematical model was derived to determine how the linear guide arrangement affects the dynamic characteristics of the stage. Results of the modal analysis show that the vibration behaviors of the positioning stage are dominated by the rigidity of the linear components and the platform. In addition, comparisons of the results from the two approaches further indicate that the platform rigidity is an important factor determining the accuracy of the prediction of the vibration frequencies by the analytically mathematical model. As a conclusion of the study, the analytically mathematical model can approximate well to the finite element model when the linear stage is designed with appropriate structure rigidity.
\end{abstract}

\section{Introduction}

In recent years, high precision positioning systems constructed of various linear rolling components have been widely used in the field of CNC machine tool, semiconductor manufacturing equipment, or inspection apparatus. In these linear feeding stages, there exist rolling interfaces between the rolling balls and the raceways of the linear components. Essentially, the bearing stiffness of the rolling elements is determined by the preloaded state of the rolling balls and has been shown to have significant influences on the dynamic characteristics of the stage [1-5]. According to the study of Lai and Hung [6], the feeding stage demonstrated different dynamic behavior when it was constructed into different configurations with linear components of different specifications. On the other hand, the milling tool showed different machining performances, varying with the preloaded amount of the feeding stage of spindle head $[7,8]$. Study of Kolar et al. [8] further addressed the fact that the dominant vibration behaviors of the spindle tool were partly determined by the construction of the feeding system, apart from its dynamics. Therefore, gaining accurate prediction of the dynamic characteristics is of importance for the design of the feeding system to achieve the desired performance.

Evaluation and optimization of a machining system with different configurations are complex processes in the design phase. Therefore, virtual prototyping technology is considered as a promising approach to avoid the traditionally timeand cost-consuming development process $[9,10]$. With an iterative process in meeting the performance requirements, the feeding or positioning system of a machine tool or automatic apparatus can be analyzed and redesigned. However, the prototyping technology requires rapid and accurate 
assessment of the dynamic characteristics of the designed system with all possible design configurations. Generally, an adequate description of the dynamic behavior of a complex mechanical structure can be derived by finite element analysis (FEA) or multibody simulation (MBS) $[9,11]$. The former method is widely used for predicting structural static or dynamic behaviors under various loading conditions and for optimizing the structural components in the detailed design phase [12]. The multibody simulation in terms of analytically derived model extends the possibilities for analyzing the motion dynamic characteristics in the time domain taking into account the behavior of the control loops of the drivers $[13,14]$.

In finite element method, the machine structure with linear components can also be appropriately modeled with sufficient accuracy by introducing the modeling schemes of the rolling elements into the analysis model $[6,7]$. However, creation of the finite element models for all structure components is a complicated task, especially when the variation in dynamic characteristics with the change in configuration of the structure modules is concerned [11, 15]. The analytically mathematical model is derived based on the assumption that the structural modulus or components are considered as rigid bodies, instead of being considered as the flexible or elastic structures in finite element model. It follows that the analytical model cannot adequately reflect the dynamic characteristics of a real machine, hence leading to inaccurate modeling results, whereas, considering the efficiency in analysis in the initial design phase, development of a simplified analytically mathematical model integrated with dimensional parametric design concept for the machine structure system is necessary. This can be an effective method to help the designer to determine the candidate model from a set of possible designs.

This study was aimed at modeling the dynamic behaviors of the linear driven positioning or feeding stages by means of the finite element analysis and analytical modeling approaches. For this purpose, different finite element models of the stages with different linear components were created for modal analysis. In order for the analysis to be realistic, the interface characteristics existing between the rolling elements and the ball grooves or raceways were accurately described based on Hertzian contact theory. Then, the analytical model was proposed to serve as an alternative tool to rapidly evaluate the influence of the design change or the arrangement of the linear components on the dynamic characteristics of a positioning stage. The applicability of analytical model was validated by the simulation results of finite element modeling of the stages designed with different structure properties.

\section{Finite Element Modeling Approach}

2.1. Construction and Specification of the Stage Prototype. A typical feed drive servomechanism for precision positioning, such as those found in machine tools, is usually constructed of machine base, planar platform or table, and linear driven mechanisms. The platform or table is supported by the carriages or slide blocks on linear guide pairs and driven with ball screw/nut mechanism, as shown in Figure 1(a). Generally, the linear guide modulus can be arranged into different configurations that may bring the stage to show a different dynamic behavior. To examine the variation of the dynamic characteristics due to the linear guides in different arrangements, a simplified small scale stage model was designed for analysis, as shown in Figure 1(b). The planar platform was horizontally supported by four slide blocks on two guide rails, which could be secured on the base, in parallel, at span of 200 or $280 \mathrm{~mm}$. The commercial linear guide blocks have four ball grooves formed as circular arc profiles with contact angle of 45 degree and are preloaded at low amount $(\mathrm{Z} 0,0.00 \sim 0.02 C)$, where $C$ is the dynamic load capacity $(7.83 \mathrm{kN})$. The driven ball screw has a diameter of $20 \mathrm{~mm}$ and a basic dynamic load capacity $C$ of $4.07 \mathrm{kN}$ with a preload of $0.06 C$. Standardized ball screw support units were used at both ends of the screw shaft.

\subsection{Modeling of Linear Components with Rolling Elements.} For linear components, such as ball screw/nut and linear guides, the rolling interfaces between rolling balls and grooves or raceways primarily contribute to the structural dynamic characteristics of the feeding stage [16]. Therefore, modeling of the rolling interface is the prerequisite for the creation of the analysis model to achieve accurate evaluation of the dynamic characteristics of a feeding system. As shown in Figure 2, the linear guide was designed with four rows of rolling balls, where the ball groove had the contact profile of a circular arc, forming a two-point contact mode [17]. The ball screw was designed with a Gothic groove, forming a fourpoint contact mode.

In general, the contact force between a rolling ball and the raceway can be related to the local deformation at the contact point by the Hertzian expression [18]:

$$
Q=K_{h} \delta^{3 / 2}
$$

where $Q$ denotes the contact force and $\delta$ is the elastic deformation at the contact point. $K_{h}$ represents the Hertz constant, which is determined by the contact geometrical dimensions of the ball groove or raceway and the material properties of the contacting components. The details are available in the literature $[19,20]$ and described as follows:

$$
\begin{gathered}
K_{h}=\frac{\pi k E^{\prime}}{3 f} \sqrt{\frac{2 C \varepsilon}{f}}, \\
k=1.0339\left(\frac{C_{y}}{C_{x}}\right)^{0.6360}, \\
f=1.5277+0.6032 \ln \left(\frac{C_{y}}{C_{x}}\right), \\
\varepsilon=1.0003+0.5968\left(\frac{C_{x}}{C_{y}}\right), \\
\frac{2}{E^{\prime}}=\frac{1-\mu_{a}^{2}}{E_{a}}+\frac{1-\mu_{b}^{2}}{E_{b}} .
\end{gathered}
$$




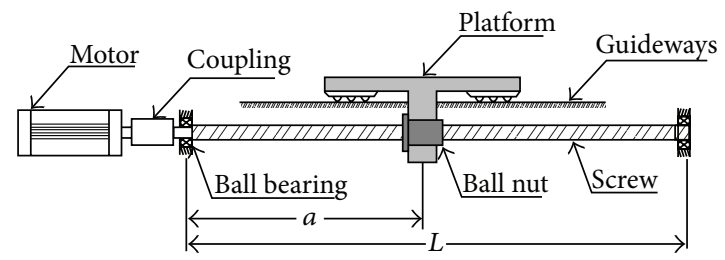

(a)

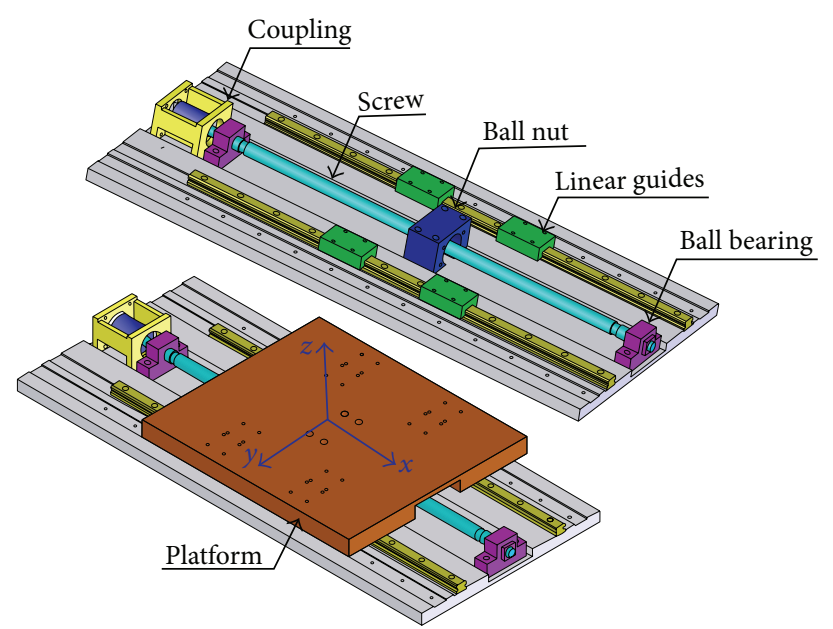

(b)

Figure 1: (a) A typical feed drive servomechanism for precision positioning; (b) a simplified small scaled stage.

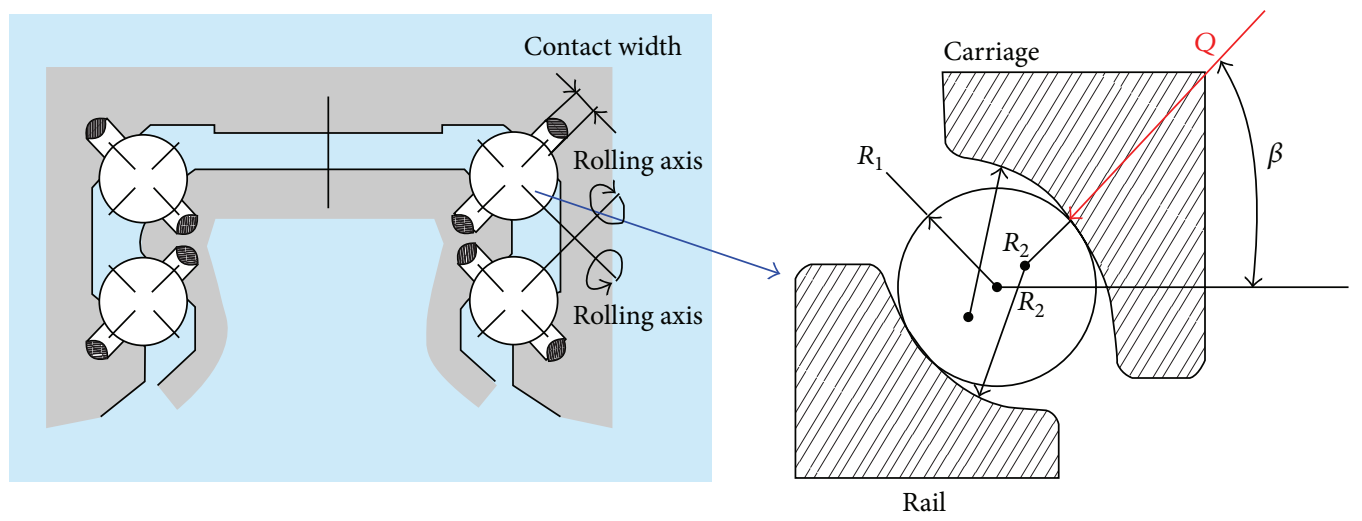

(a) Schematic of contact geometry of a rolling guide

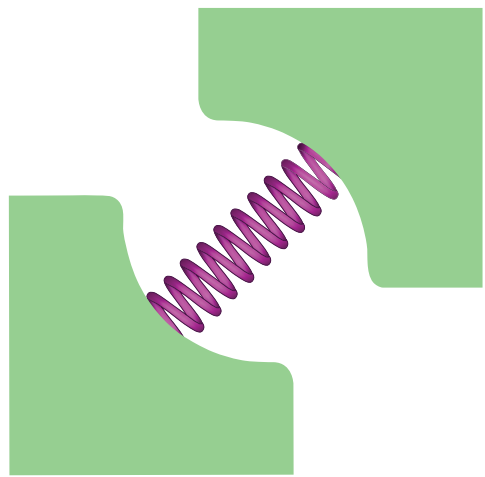

(b) Modeling of linear rolling guide

FIGURE 2: Schematic of contact geometry of a rolling guide with four rows of rolling balls, forming a two-point contact mode within each ball groove. The contact mode can be regarded as a type of Hertzian contact mode. In the figure, $\beta$ and $Q$ represent the contact angle and contact force, and $R_{1}$ and $R_{2}$ are the radii of rolling ball and ball grooves, respectively. (b) Modeling of linear rolling guide.

In the above equations, $E$ represents Young's modulus; $\mu$ denotes Poisson's ratio of the material; and constants $C, C_{x}$, and $C_{y}$ are related to the diameter $D$ of the rolling ball and the radius $R_{i}$ of the raceway, which can be expressed as shown in the following equation, in terms of the form factor $f_{i}$ of the contact geometry of the raceway:

$$
C_{x}=\frac{D}{2}, \quad C_{y}=D \frac{f_{i}}{2 f_{i}-1}, \quad C=\frac{C_{x} C_{y}}{C_{y}-C_{x}} .
$$




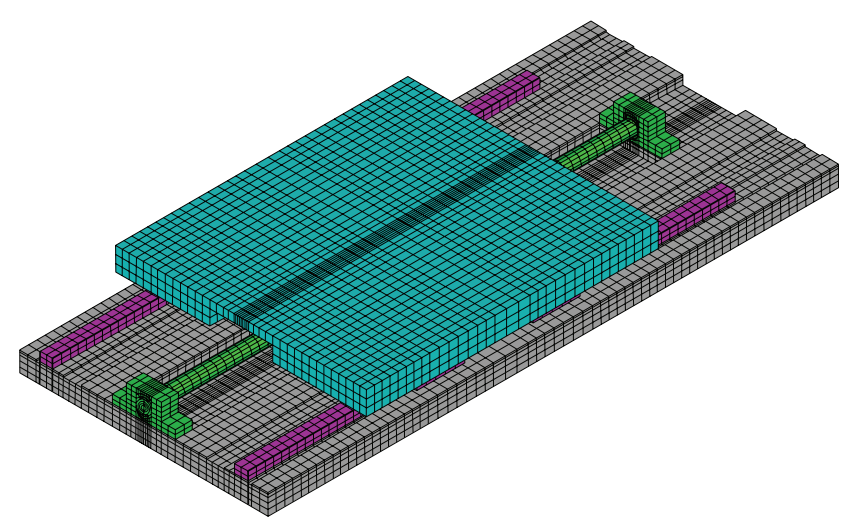

(a)

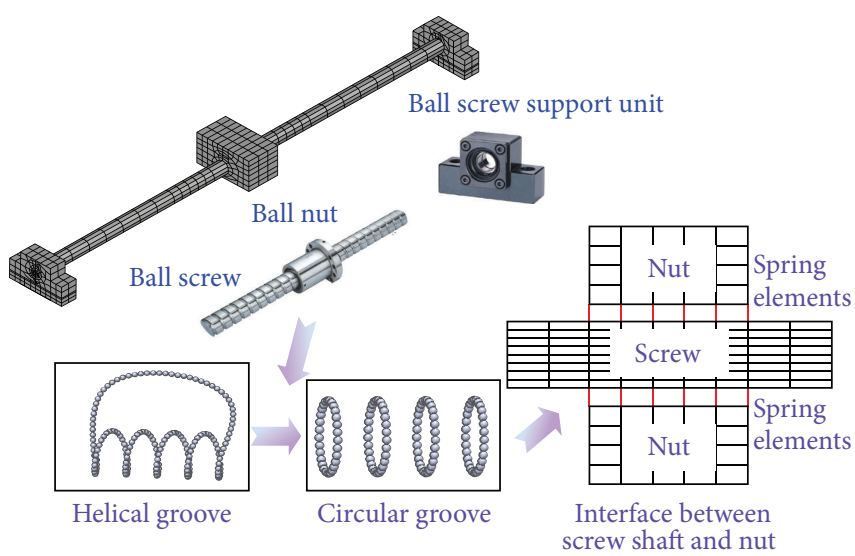

(b)

FIGURE 3: (a) Finite element model of the positioning stage; (b) modeling of ball screw and ball bearings.

The normal stiffness at a specific preload can then be obtained as

$$
K_{n}=\frac{d Q}{d \delta}=\frac{3}{2} K_{h} \delta^{1 / 2}=\frac{3}{2} K_{h}^{2 / 3} Q^{1 / 3}
$$

As revealed in (4), the contact stiffness in the normal direction is affected by the contact force; hence, the external loads imposed on the carriage block will alter the bearing stiffness of the rolling guide to deviate from the initial value that is determined by the initial preload set on the rolling ball. It can therefore be expected that the dynamic characteristic will vary with the loads acting on the positioning stage with rolling guides.

2.3. Finite Element Model of Stage. The finite element model of the feeding stages with driven ball screw mechanism was created for modal analysis, as shown in Figure 3, in which the guide rails were arranged at a span of 200 and $280 \mathrm{~mm}$, respectively. The components of the driven mechanism in a positioning stage, such as ball screw, ball nut, and linear guides, were also included in the models. For the creation of the finite element model with linear components, the modeling criteria well validated in previous study $[6,7]$ were followed. The main bodies of the linear components were modeled as solid elements and connected with spring elements at the rolling interfaces by intentionally ignoring the effect of the rolling balls, but the spring elements at each ball groove had an overall contact stiffness equivalent to that of the original guide model.

The stage model with driven mechanism was meshed with brick elements of 23242 elements and 31105 nodes. Based on the main specifications of the linear guide modules (ball diameter $d=2.778 \mathrm{~mm}$, form factor $=0.52$, and dynamic loading capacity $C=7830 \mathrm{~N}$ ), the contact stiffness $K_{n}$ at contact point was calculated as $8.7 \mathrm{~N} / \mu \mathrm{m}$ for low preloaded rolling balls.

In a similar way, the contact mode of the rolling balls between ball screw and ball nut was simplified as circular contact pairs with the introduction of spring elements at the rolling interface. The overall stiffness was estimated to be $152 \mathrm{~N} / \mu \mathrm{m}$ according to the specifications of the ball screw [21]. As to the bearing, the inner and outer rings were also connected by spring elements distributed around the ring raceway, which provided the stiffness in both axial and radial directions to sustain the ball screw. The bearing stiffness of the angular contact bearing was obtained from the bearing manufacturer, which was reported to be $88 \mathrm{~N} / \mu \mathrm{m}$ [22].

For finite element analysis, the material used for all components was carbon steel with an elastic modulus $E=$ $200 \mathrm{GPa}$, Poisson's ratio $\mu=0.3$, and density $\rho=7800 \mathrm{Kg} / \mathrm{m}^{3}$. The shapes of the vibration mode associated with the frequencies of the stage were obtained by implementing the modal analysis into the finite element computations.

\section{Analytical Modeling Approach}

3.1. Model Description. In mathematical modeling, the stage system was considered as the mass spring system with multiple degrees of freedom. The planar platform was modeled as a rigid plate supported by various spring elements simulating the different linear components. The coordinate system for vibration motions of the stage is illustrated in Figure 4, where the origin of the coordinate is located at the mass center of the platform. The sliding direction of the platform is along the $x$ axis of rail guide, $\beta$ is the contact angle between the rolling ball and the raceway in linear guide modulus, $d$ and $e \pm e_{0}$ are the distances from the contact point between the rolling ball and carriage to the $x-y$ plane and the $x-z$ plane, respectively, and $l$ is the distance from the mass center of the platform to the $y$ - $z$ plane.

For such a spring mass system, we can define the vibration mode associated with the motion degrees of freedom. As shown in Figure 4, the main transverse motions $u$ and $v$ are defined as linear displacement in horizontal and vertical directions, respectively, in which the displacement in vertical direction is called vertical vibration mode. The rocking motion $\theta$ about the $y$-axis is termed the pitching vibration mode. The rocking motion $\psi$ about the $z$-axis is the yawing 


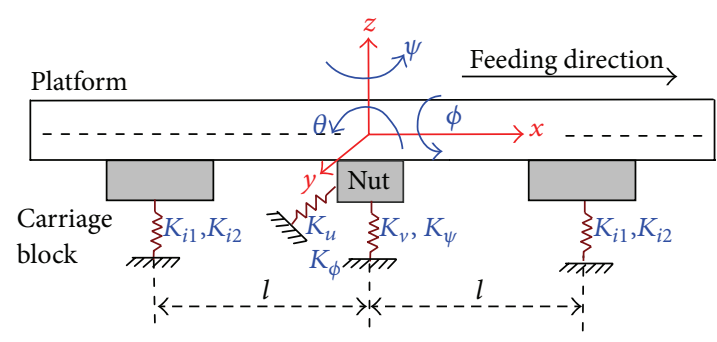

(a)

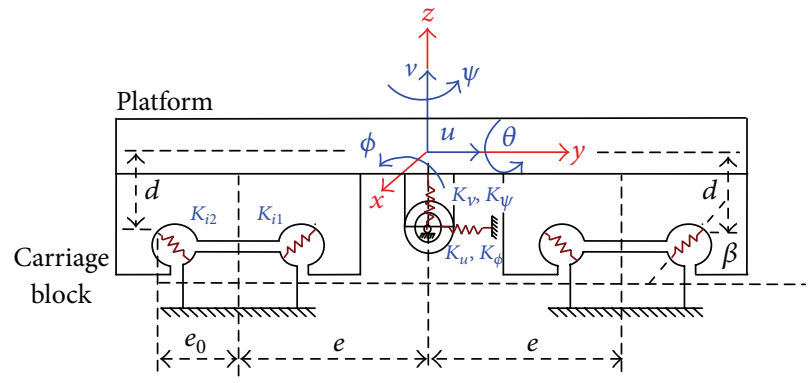

(b)

FIgURE 4: Schematic of the dynamic structural model of the ball screw driven stage with linear rolling guides.

vibration mode and the rocking motion $\phi$ about the $x$-axis is the rolling vibration mode.

The linear guide modulus supporting the platform was simulated by linear springs with adequate stiffness that was determined by their preload condition based on the Hertzian contact theory, as discussed in previous section. The rigidity of the linear guide modulus is governed by the contact stiffness of the rolling interface between the rolling ball and raceway because the guide rail and carriage block are more rigid in structure. Therefore, by neglecting rolling balls and rail, each guide module was modeled by two spring elements, acting along the direction of the contact angle at each ball groove, respectively. The corresponding contact stiffness was, respectively, quantified with the stiffness $K_{i 1}$ and $K_{i 2}$ for each guide module $(i=1,4)$, Figure 4 . Similarly, the ball screw/nut mechanism with the supporting bearings at both ends was considered as a horizontal beam with fixed ends and was mounted on the center of the platform. Considering the ability to resist the lateral force and bending moment applied on the stage, the screw shaft was simulated by two pairs of springs in vertical and horizontal direction, respectively, which can be further described by stiffness matrix corresponding to the degrees of freedom $u, v, \theta$, and $\psi:$

$$
[K]=\left[\begin{array}{ll}
K_{u} & K_{\theta} \\
K_{\psi} & K_{v}
\end{array}\right],
$$

where $K_{v}$ and $K_{u}$ are the bending stiffness of the ball screw against the transverse force along the $z$-axis and the $y$-axis, respectively. $K_{\theta}$ and $K_{\psi}$ are the rotational stiffness of the ball screw against the bending moment about the $y$-axis and the $z$-axis, respectively. Based on Timoshenko's beam theory, the bending and rotational stiffness are expressed as follows:

$$
\begin{gathered}
K_{v}=K_{u}=\frac{3 E I L^{3}}{a^{3}(L-a)^{3}}, \\
K_{\theta}=K_{\psi}=\frac{4 E I L}{\left(3 a^{2}-L a\right)},
\end{gathered}
$$

where $E$ and $I$ are Young's modulus and the moment of inertia of the screw shaft. $L$ is the screw length and $a$ is the location of the platform mounted with the ball nut on screw shaft.
3.2. Formula of Natural Frequency. The governing equations of the five degrees of freedom model were derived in terms of the application of Lagrange's approach to the potential energy of the platform-spring system. Detailed derivations were listed in the appendix. The natural frequencies of the vibration of the stage platform including the yawing mode $\left(f_{Y}\right)$, pitching mode $\left(f_{P}\right)$, vertical mode $\left(f_{V}\right)$, lower rolling mode $\left(f_{\mathrm{RL}}\right)$, and higher rolling mode $\left(f_{\mathrm{RH}}\right)$ are shown as follows:

$$
\begin{aligned}
& f_{Y}=\frac{1}{2 \pi} \sqrt{\frac{8 K l^{2} \cos ^{2} \beta+K_{\psi}}{J_{z}}}, \\
& f_{V}=\frac{1}{2 \pi} \sqrt{\frac{8 K \sin ^{2} \beta+K_{V}}{M}}, \\
& f_{P}=\frac{1}{2 \pi} \sqrt{\frac{8 K l^{2} \sin ^{2} \beta+K_{\theta}}{J_{y}}}, \\
& f_{\mathrm{RL}}=\frac{\omega_{1}}{2 \pi}, \quad f_{\mathrm{RH}}=\frac{\omega_{2}}{2 \pi}
\end{aligned}
$$

where

$$
\begin{gathered}
\omega_{1,2}^{2}=\frac{c_{3} M+c_{1} J_{y} \mp \sqrt{\left(c_{3} M+c_{1} J_{x}\right)^{2}-4 M J_{x}\left(c_{1} c_{3}-c_{2}^{2}\right)}}{2 M J_{x}}, \\
C_{1}=8 K \cos ^{2} \beta+K_{u}, \\
C_{2}=-8 K d \cos ^{2} \beta-8 K e_{0} \sin \beta \cos \beta \\
C_{3}=8 K\left(d \cos \beta+e_{0} \sin \beta\right)^{2}+8 K e^{2} \sin ^{2} \beta
\end{gathered}
$$

In (7)-(8), $M$ is the mass of the platform; $J_{x}, J_{y}$, and $J_{z}$ are the moments of inertia of the platform about the $x$-axis, $y$-axis, and $z$-axis, respectively; $K$ is the contact stiffness of the linear guide modulus. Related parameters required for solutions of the frequencies are listed in Table 1. 
TABLE 1: Parameters of the ball screw driven stage.

\begin{tabular}{lcc}
\hline Parameter & Value & Unit \\
\hline Total stage mass, $M$ & 36.866 & $\mathrm{~kg}$ \\
Moments of inertia, $J_{x}, J_{y}, J_{z}$ & $0.45085,0.5115,0.95064$ & $\mathrm{Kg} \mathrm{m}$ \\
Geometry dimensions, & 140 & $\mathrm{~mm}$ \\
$e_{1}=e_{2}=e_{3}=e_{4}=e$ & 83.5 & $\mathrm{~mm}$ \\
$l_{1}=l_{2}=l_{3}=l_{4}=l$ & 27.962 & $\mathrm{~mm}$ \\
$d_{1}=d_{2}=d_{3}=d_{4}=d$ & $20,700,350,10.5$ & $\mathrm{~mm}$ \\
$D, L, a, e_{0}$ & $139.2 e^{6}$ & $\mathrm{~N} / \mathrm{m}$ \\
Spring constant (linear guide) & & \\
$K=K_{i 1}=K_{i 2}(i=1,4)$ & $0.879 e^{6}$ & $\mathrm{~N} / \mathrm{m}$ \\
Spring constant (ball screw) & & \\
$K_{v}=K_{u}$ & $0.0359 e^{6}$ & $\mathrm{~N}-\mathrm{m} / \mathrm{rad}$ \\
Spring constant (ball screw) & & \\
$K_{\theta}=K_{\psi}$ &
\end{tabular}

\section{Results and Discussions}

4.1. Vibration Modes of the Stages. The first five vibration mode shapes of the positioning stage predicted by modal analysis are shown in Figure 5. It is obvious that these vibration motions are closely governed by the fundamental vibration modes of the carriage blocks on guide rails, which are termed yawing, pitching, and rolling motions $[6,17]$. The first vibration mode of the stage is the yawing vibration of the platform about the vertical axis ( $z$-axis), which is dominated by the yawing motion of the carriage blocks of the linear guides. The second mode is the pitching vibration of the platform about the $y$-axis, dominated by the in-phase pitching motions of the linear guide blocks. The third mode is associated with rolling vibration of platform about the $x$ axis, also dominated by the rolling motion of the carriage blocks on the linear guides. The fourth mode is the vibration of the platform in vertical direction, mainly caused by the carriage blocks in out-of-phase pitching motion and partly accompanied by the bending of the platform about $y$-axis. The fifth mode is the deformed platform vibrating in rolling motion at higher frequency about the $x$-axis. It is observed from mode shapes that the vibration motions of the platform are also partly accompanied by the bending deformation of the plate, especially at the higher modes of rolling and vertical motions. Therefore, we can realize that natural frequencies associated with these vibration modes are determined by the bearing stiffness of the linear components and the structure rigidity of the platform.

\subsection{Vibration Frequencies of the Stage}

4.2.1. Finite Element Predictions. The fundamental vibration frequencies of linear stage predicted from finite element modal analysis are listed in Table 2, which also compares the differences between the stages with guide span of 200 and $280 \mathrm{~mm}$. As indicated in Table 2, there is no significant difference in frequencies associated with the yawing and pitching modes for stages with different guide spans. However, the vibration frequency of the vertical mode increases by approximately $12.6 \%$, when the guide span is changed from 200 to $280 \mathrm{~mm}$, while the lower and higher rolling vibration modes show a reduction of $18 \%$ and $11 \%$ in vibration frequency, respectively. Results of the modal analysis clearly illustrate that the span of the linear guide pairs affects the vibration characteristics of the stage to different extent, varying with the vibration modes. As suggested in the technical specifications, the linear guides could be mounted on the machine base in various orientations according to the design requirements. However, the bearing stiffness of the linear guide module was shown to vary with the applied loads although the contact geometry was designed to withstand loads from different directions $[6,23]$. Therefore, the influence of the mounting orientation of the rolling guides is worth further discussion, apart from the effect of the guide span that was discussed in the study.

4.2.2. Analytical Results. The natural frequencies associated with the fundamental vibration modes of the stage were calculated by substituting the parameters in Table 1 into the mathematical models. Table 3 compares the natural frequencies for stage with guide span of 200 and $280 \mathrm{~mm}$. As revealed in Table 3, there is no significant difference in the frequencies associated with the yawing, pitching, and vertical modes, but the lower and higher rolling vibration modes are greatly affected by guide span, resulting in a reduction of frequency by $18.03 \%$ approximately when the guide span is changed from 200 to $280 \mathrm{~mm}$. As was found in finite element predictions, analytical results also verify the effect of the guide span on the vibration frequencies of the feeding stage.

The natural frequencies predicted by finite element simulation and analytical calculation are further compared in Figure 6. It is found that the frequencies calculated from analytical model are higher than those predicted by finite element model. The frequency differences between the two models become more significant at higher modes. This implies that the analytical model derives higher structure stiffness as compared with the finite element model. A known fact is that the structure stiffness of the feeding stage is contributed by the bearing stiffness of linear components and rigidity of the platform. In the analytical model, the platform was assumed to be rigid, having infinite stiffness. Only the elastic deformation of the contact interface was considered in the analytical calculations. However, in finite element modeling, the stage was assumed as an elastic solid structure supported by the linear springs with different stiffness. This makes the finite element model possess a lower structural stiffness than the analytical model. Besides, according to the mode shapes illustrated in Figure 5, the platform also undergoes elastic bending or twisting deformation when it vibrates under the elastic constraints of the linear components. This further means that the flexibility of the platform also contributes to the vibration behavior to some extent. In addition, such effects become more significant when the platform vibrates at higher modes, such as rolling and vertical motions.

Comparisons of the results give the implication that the inconsistence in predicting the vibration frequency between 

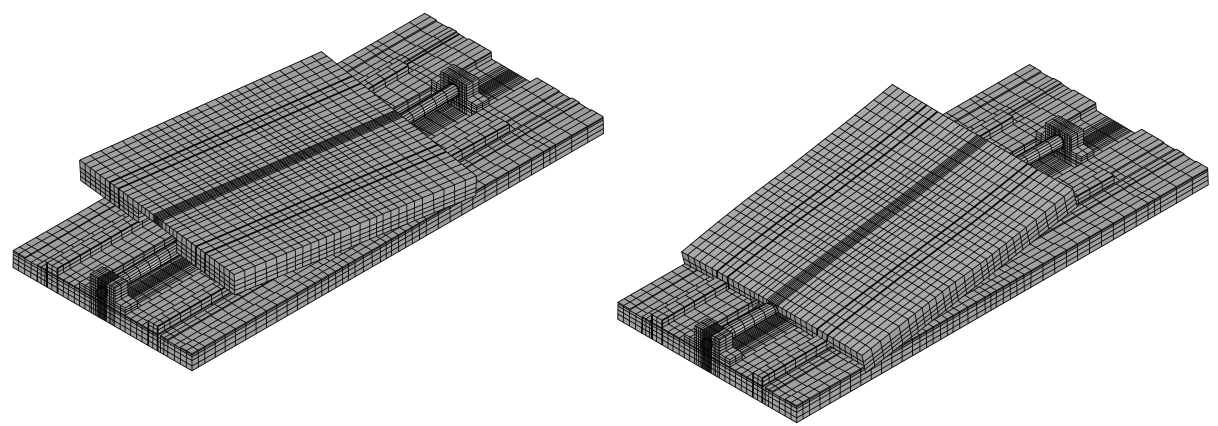

Yawing mode

Pitching mode
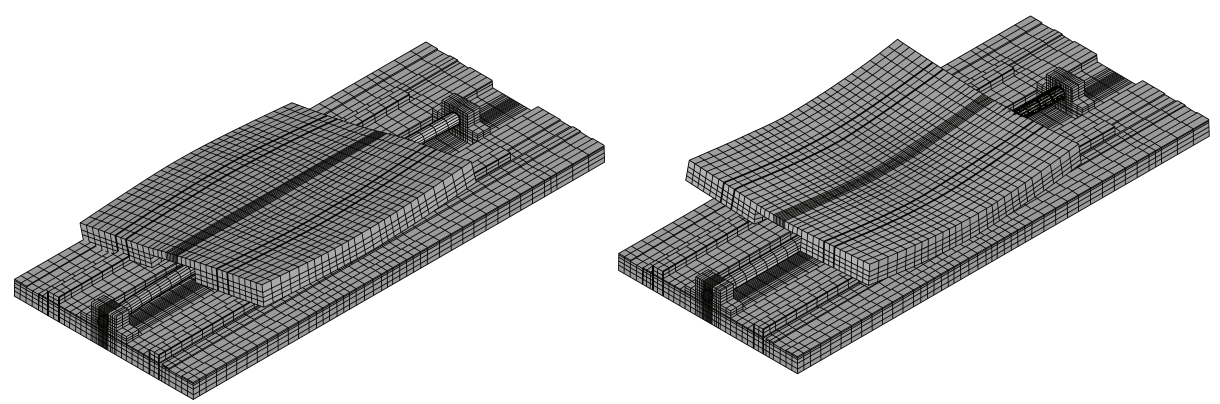

Lower rolling mode

Vertical mode

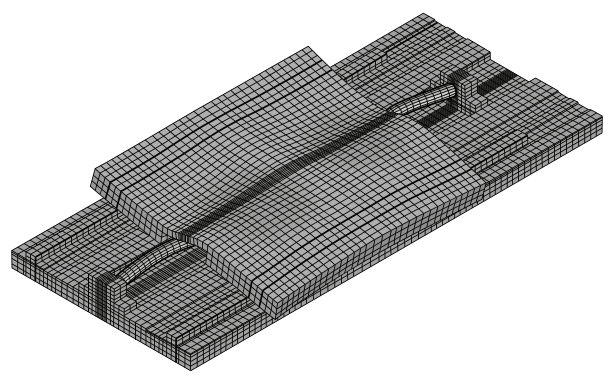

Higher rolling mode

FIGURE 5: The first five vibration mode shapes of the positioning stage.

TABLE 2: Modal frequencies of the feeding stage predicted by finite element approach.

\begin{tabular}{lccccc}
\hline Stage model & & \multicolumn{4}{c}{ Vibration mode } \\
& Yawing mode & Pitching mode & Lower rolling mode & Vertical mode & Higher rolling mode \\
\hline Guide span $(200 \mathrm{~mm})$ & 323 & 459 & 477 & 604 & 677 \\
Guide span $(280 \mathrm{~mm})$ & 322 & 444 & 563 & 528 & 784 \\
\hline Difference $(\%)$ & 0.4 & 3.3 & 18.0 & 12.6 & 11.4 \\
\hline
\end{tabular}

TABLE 3: Modal frequencies of the feeding stage predicted by analytical approach.

\begin{tabular}{lccccc}
\hline Stage model & & \multicolumn{4}{c}{ Vibration mode } \\
& Yawing mode & Pitching mode & Lower rolling mode & Vertical mode & Higher rolling mode \\
\hline Guide span $(200 \mathrm{~mm})$ & 343 & 468 & 517 & 657 & 754 \\
Guide span $(280 \mathrm{~mm})$ & 341 & 468 & 608 & 657 & 885 \\
\hline Difference $(\%)$ & 0.7 & 0.0 & 17.5 & 0.0 & 17.3 \\
\hline
\end{tabular}




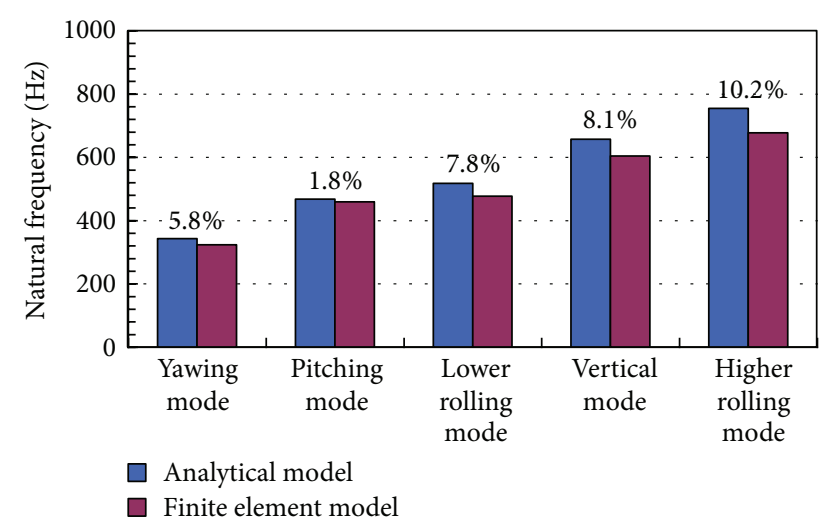

FIGURE 6: Comparisons of the frequencies of the stage predicted by finite element simulation and analytical calculation.

the analytically mathematical model and the finite element model is determined by the relative rigidity between the platform and the linear component.

4.3. Applicability of Analytical Model: Effect of the Stage Rigidity. Based on the above analysis, we could understand that structure rigidity of stage platform plays an important role in deciding whether the analytical model can approach the finite element model. To realize the applicability of proposed analytical mode, in this section, we created another three stage models by thickening the planar platform from original model by a ratio of 20,30 , and $50 \%$, respectively. On the other hand, since the modal stiffness is one measure of the ability of the structure to resist the deformation under a specific resonance mode vibration of the structure, which also contributes to a part of the overall structure, we further defined the rigidity ratio as the ratio of the lowest modal stiffness of the platform to the bearing stiffness of the linear components of the stage.

With the rigidity ratio, we can effectively determine the structure component that dominates the overall structural behavior of the stages. The rigidity ratio of the four stage models was calculated as $0.41,1.03,2.40$, and 4.02 , respectively. The natural frequencies of these different stages were further estimated by analytical calculation and finite element simulation, respectively. According to the results in Table 4, the stage with thicker platform behaves lower frequencies than that with thinner platform. This can be ascribed to the fact that the increased mass of the thicken platform shows more influence on the natural frequency than the increment of the structure rigidity.

To get clear insight into the effect of the rigidity ratio, the natural frequencies predicted by the two methods for different stages are depicted in Figures 7 and 8 for guide spans of 200 and $280 \mathrm{~mm}$, respectively. As shown in Figure 7, the two models predicted the variation of vibration frequency in a similar tendency but had discrepancy between them. For example, in the case of the stage platform with rigidity ratio of 0.41 (case 1), the differences in modal frequencies predicted by analytical and finite element approaches are around 5.78\%

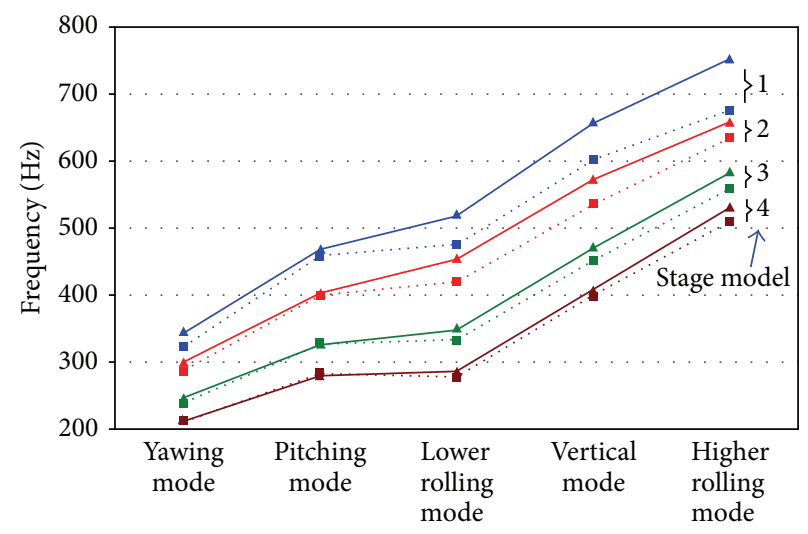

Figure 7: Comparison of the natural frequencies of the stages between the finite element simulation (solid line) and the analytical calculation (dotted line). The four stage models (1 to 4 ) are rated with different structure rigidity ratios as $0.40,1.03,2.40$, and 4.02, respectively (guide span $=200 \mathrm{~mm}$ ).

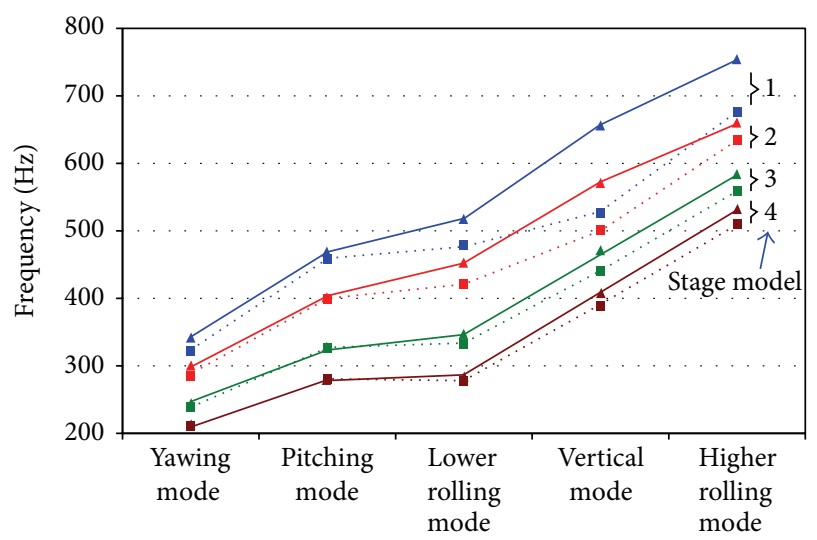

FIGURE 8: Comparison of the natural frequencies of the stages between the finite element simulation (solid line) and the analytical calculation (dotted line). The four stage models (1 to 4) are rated with different structure rigidity ratios as $0.40,1.03,2.40$, and 4.02, respectively (guide span $=280 \mathrm{~mm}$ ).

at lower modes and $10.21 \%$ at higher modes. For stage cases 2,3 , and 4 with rigidity ratios of $1.03,2.40$, and 4.02 , the maximum difference between the results predicted by the two approaches is less than 5\%, occurring at higher rolling modes. Consistent trend is also found in modeling the stage with guide span of $280 \mathrm{~mm}$. The maximum difference between the two simulations is found to occur at the vertical mode, about $12-19 \%$, for the stage with rigidity ratios of 0.41 and 1.03. The great difference can be ascribed to the fact that a stage platform with lower rigidity and supported with wider span shows more significant bending deformation during vertical vibration. This phenomenon has been demonstrated in the finite element model; however, it was not involved in the analytical model. Meanwhile, the difference between the two different estimations is found to lessen when the platform structure of stage is appropriately enhanced with higher rigidity. 
TABLE 4: Natural frequencies of the stage with different rigidity ratios predicted by analytical calculation and finite element simulation.

\begin{tabular}{|c|c|c|c|c|c|c|}
\hline Rigidity ratio of stage & Method & Yawing mode & Pitching mode & Lower rolling mode & Vertical mode & Higher rolling mode \\
\hline \multirow{3}{*}{0.41} & Analytical analysis & 343.2 & 467.9 & 517.3 & 657.3 & 754.3 \\
\hline & $\begin{array}{l}\text { Finite element } \\
\text { analysis }\end{array}$ & 323.3 & 459.3 & 477.2 & 604.1 & 677.3 \\
\hline & Difference & $5.78 \%$ & $1.83 \%$ & $7.76 \%$ & $8.10 \%$ & $10.21 \%$ \\
\hline \multirow{3}{*}{1.03} & Analytical analysis & 299.1 & 403.0 & 452.7 & 572.2 & 659.6 \\
\hline & $\begin{array}{c}\text { Finite element } \\
\text { analysis }\end{array}$ & 287.8 & 401.7 & 420.1 & 536.9 & 634.4 \\
\hline & Difference & $3.78 \%$ & $0.33 \%$ & $7.21 \%$ & $6.17 \%$ & $3.82 \%$ \\
\hline \multirow{3}{*}{2.40} & Analytical analysis & 245.8 & 325.8 & 346.1 & 469.6 & 584.8 \\
\hline & $\begin{array}{c}\text { Finite element } \\
\text { analysis }\end{array}$ & 240.3 & 328.1 & 334.3 & 452.8 & 559.2 \\
\hline & Difference & $2.25 \%$ & $0.71 \%$ & $3.40 \%$ & $3.57 \%$ & $4.37 \%$ \\
\hline \multirow{3}{*}{4.02} & Analytical analysis & 213.6 & 279.6 & 286.3 & 407.7 & 531.1 \\
\hline & $\begin{array}{l}\text { Finite element } \\
\text { analysis }\end{array}$ & 210.0 & 282.2 & 277.7 & 395.5 & 507.5 \\
\hline & Difference & $1.69 \%$ & $0.92 \%$ & $3.02 \%$ & $2.99 \%$ & $4.44 \%$ \\
\hline
\end{tabular}

As observed from analysis results, the analytical model predicted results deviate more from the finite element solutions for stage with the low rigidity ratio, but the differences between them decrease with the increasing in rigidity ratio of the stage. As was defined, a stage with high rigidity ratio means that the platform of the stage is more rigid than the supporting components. If a stage is designed with enough rigidity ratios, the linear components will give more influence on the vibration characteristics of the stage than the structure rigidity of platform. In other words, the influence of the platform structure on the overall structure stiffness of the stage gradually lessens with the increasing rigidity ratio. Such phenomenon has been verified in the analytical models of the sage with a rigidity ratio higher than 2.4 , which shows the predicted vibration frequencies approaching those obtained from finite element models.

Basically, in derivation of the analytically mathematical model, the machine structure was assumed as rigid bodies mounted on elastic supports of different types. This makes the analytical model unable to stand for a real machine structure accurately, hence resulting in a greater deviation from the finite element model in predicting the vibration characteristics. However, current analysis results obviously suggest that the analytically mathematical model derived for a linear stage designed with higher rigidity ratio in structure configuration can approximate well with the finite element model. This also gives an implication that the analytical approximation could be an effective tool in modeling the dynamic behavior of a feeding system when it was constructed with adequate rigidity of the structure modulus and linear components.

\section{Conclusions}

In this study, the dynamic behaviors of the feeding stages constructed with different arrangements of linear guides were analyzed by means of the finite element analysis and analytical modeling approaches. According to the finite element modal analysis, it is found that the dynamic behaviors of the stage platform are mainly governed by the fundamental vibration modes of the carriage blocks of linear guides and partly accompanied by the elastic deformation of the platform. As realized from this analysis, vibration characteristics are mainly determined by the bearing stiffness of the linear guide modulus and the rigidity of the platform. The dominance of the two properties can be quantified by the rigidity ratio which is defined as the ratio of the modal stiffness of platform to the bearing stiffness of linear guides. A feeding stage with high rigidity ratio means that the linear guide system dominates the dynamic behavior. On the contrary, for a stage with low rigidity ratio, the flexibility of the platform affects the dynamic behavior more than the supporting guide system.

Moreover, comparisons of the results predicted by the two approaches show that the rigidity ratio of the stage platform is an important factor affecting the accuracy in modeling the vibration behavior based on the analytically mathematical model. Conclusions of this study suggest that the analytically mathematical model approximates well to the finite element models in investigating the dynamic behavior of a stage which is designed with appropriate structure rigidity.

\section{Appendix}

Using the notation as described in Section 3.1 and referring to Figure 4, the total kinetic energy $E_{K}$ of the simplified platform model of the feeding stage can be expressed as

$$
E_{K}=\frac{1}{2} M \dot{u}^{2}+\frac{1}{2} M \dot{v}^{2}+\frac{1}{2} J_{x} \dot{\phi}^{2}+\frac{1}{2} J_{y} \dot{\theta}^{2}+\frac{1}{2} J_{z} \dot{\psi}^{2}
$$


The potential energy $E_{p}$ is contributed by all linear spring elements and can be given by

$$
\begin{gathered}
E_{P}=\sum_{i=1}^{4} \frac{1}{2}\left(K_{i 1} \delta_{i 1}^{2}+K_{i 2} \delta_{i 2}^{2}\right)+\frac{1}{2} K_{v} v^{2}+\frac{1}{2} K_{u} u^{2} \\
+\frac{1}{2} K_{\theta} \theta^{2}+\frac{1}{2} K_{\psi} \psi^{2} \\
=\sum_{i=1}^{4} \frac{1}{2}\left[K_{i 1}\left(A_{i 1} \cos \beta+B_{i 1} \sin \beta\right)^{2}\right. \\
\left.+K_{i 2}\left(A_{i 2} \cos \beta+B_{i 2} \sin \beta\right)^{2}\right]+\frac{1}{2} K_{v} v^{2} \\
+\frac{1}{2} K_{u} u^{2}+\frac{1}{2} K_{\theta} \theta^{2}+\frac{1}{2} K_{\psi} \psi^{2},
\end{gathered}
$$

where

$$
\begin{array}{ll}
A_{11}=u-d_{1} \phi+l_{1} \psi, & B_{11}=-v-\left(e_{1}+e_{0}\right) \phi+l_{1} \theta, \\
A_{12}=-u+d_{1} \phi-l_{1} \psi, & B_{12}=-v-\left(e_{1}-e_{0}\right) \phi+l_{1} \theta, \\
A_{21}=u-d_{2} \phi+l_{2} \psi, & B_{21}=-v+\left(e_{2}-e_{0}\right) \phi+l_{2} \theta, \\
A_{22}=-u+d_{2} \phi-l_{2} \psi, & B_{22}=-v+\left(e_{2}+e_{0}\right) \phi+l_{2} \theta, \\
A_{31}=u-d_{3} \phi-l_{3} \psi, & B_{31}=-v-\left(e_{3}+e_{0}\right) \phi-l_{3} \theta, \\
A_{32}=-u+d_{3} \phi+l_{3} \psi, & B_{32}=-v-\left(e_{3}-e_{0}\right) \phi-l_{3} \theta, \\
A_{41}=u-d_{4} \phi-l_{4} \psi, & B_{41}=-v+\left(e_{4}-e_{0}\right) \phi-l_{4} \theta, \\
A_{42}=-u+d_{4} \phi+l_{4} \psi, & B_{42}=-v+\left(e_{4}+e_{0}\right) \phi-l_{4} \theta .
\end{array}
$$

Assume that all the linear guide moduli have the same specifications and preloaded amounts; then

$$
\begin{gathered}
e_{1}=e_{2}=e_{3}=e_{4}=e, \\
d_{1}=d_{2}=d_{3}=d_{4}=d, \\
l_{1}=l_{2}=l_{3}=l_{4}=l, \\
K_{11}=K_{12}=K_{21}=K_{22}=K_{31}=K_{32}=K_{41}=K_{42}=K .
\end{gathered}
$$

In the above equations, $\delta_{i 1}$ and $\delta_{i 2}$ are the displacement of the $i$ th carriage block supporting the platform, respectively; the subscripts 1 and 2 represent the left and right row of raceways, respectively; $d_{i}, l_{i}$, and $e_{i}$ are the distances from the contact point between the rolling ball and carriage to the $x-y$ plane, the $y-z$ plane, and the $x-z$ plane, respectively (shown in Figure 1).

Applying Lagrange's approach to (A.1) and (A.2), we can derive the motion equations with respect to different degrees of freedom:

$$
\begin{gathered}
M \ddot{u}+\left[8 K \cos ^{2} \beta+K_{u}\right] u \\
+\left[-8 K d \cos ^{2} \beta-8 K e_{0} \sin \beta \cos \beta\right] \phi=0, \\
M \ddot{v}+\left[8 K \sin ^{2} \beta+K_{v}\right] v=0, \\
J_{x} \ddot{\phi}+\left[-8 K d \cos ^{2} \beta-8 K e_{0} \sin \beta \cos \beta\right] u \\
+\left[8 K\left(d \cos \beta+e_{0} \sin \beta\right)^{2}+8 K e^{2} \sin ^{2} \beta\right] \phi=0, \\
J_{y} \ddot{\theta}+\left[8 K l^{2} \sin ^{2} \beta+K_{\theta}\right] \theta=0, \\
J_{z} \ddot{\psi}+\left[8 K l^{2} \cos ^{2} \beta+K_{\psi}\right] \psi=0 .
\end{gathered}
$$

In (A.5)-(A.9), $M$ is the mass of the platform; $J_{x}, J_{y}$, and $J_{z}$ are the moments of inertia of the platform about the $x$ axis, $y$-axis, and $z$-axis, respectively; $K$ is the contact stiffness of the linear guide modulus. The stiffness coefficients $\left(K_{v}\right.$ and $K_{u}$ ) are bending stiffness of the screw shaft against the lateral force in the $z$-axis and $y$-axis direction, and $K_{\theta}$ and $K_{\psi}$ are stiffness of the screw shaft against the bending moment about the $y$-axis and the $z$-axis, respectively, which can be calculated by the following formula:

$$
\begin{gathered}
K_{v}=K_{u}=\frac{P}{\delta}=\frac{6 E I L^{3}}{b^{2}\left[3 a L x^{2}-(3 a+b) x^{3}\right]}, \\
K_{v}=K_{u}=\frac{3 E I L^{3}}{a^{3}(L-a)^{3}} \quad(a=b), \\
K_{\theta}=K_{\psi}=\frac{4 E I L}{\left(3 a^{2}-L a\right)},
\end{gathered}
$$

where $E$ is Young's modulus, $I$ is the moment of inertia, $L$ is the length of the screw shaft, and $a$ is the location of the platform mounted with the ball nut on screw shaft.

It is noticed that (A.6) governs the displacement $v$ along the $z$-axis, (A.8) governs the angular displacement $\theta$ about $y$ axis, and (A.9) describes the angular displacement $\psi$ about $z$-axis. The natural frequencies can be obtained as calculated from these equations, namely, as follows. is

The natural frequency of the vertical vibration of the stage

$$
f_{V}=\frac{1}{2 \pi} \sqrt{\frac{8 K \sin ^{2} \beta+K_{V}}{M}} .
$$

The natural frequency of the pitching vibration of the stage is

$$
f_{P}=\frac{1}{2 \pi} \sqrt{\frac{8 K l^{2} \sin ^{2} \beta+K_{\theta}}{J_{y}}} .
$$

The natural frequency of the yawing vibration of the stage is

$$
f_{Y}=\frac{1}{2 \pi} \sqrt{\frac{8 K l^{2} \cos ^{2} \beta+K_{\psi}}{J_{z}}} .
$$


Since in (A.5) and (A.7) the displacement $u$ along the $y$ axis and the angular displacement $\phi$ about $x$-axis are mutually coupled, we may assume the solution to be of the form

$$
\begin{aligned}
u & =U e^{j \omega t}, \\
\phi & =\Phi e^{j \omega t} .
\end{aligned}
$$

Substituting (A.14) into (A.5) and (A.7), we can obtain the characteristic equation

$$
\left[\begin{array}{cc}
c_{1}-M \omega^{2} & c_{2} \\
c_{2} & c_{3}-J_{x} \omega^{2}
\end{array}\right]\left[\begin{array}{l}
U \\
\Phi
\end{array}\right]=0
$$

where

$$
\begin{gathered}
c_{1}=8 K \cos ^{2} \beta+K_{u}, \\
c_{2}=-8 K d \cos ^{2} \beta-8 K e_{0} \sin \beta \cos \beta, \\
c_{3}=8 K\left(d \cos \beta+e_{0} \sin \beta\right)^{2}+8 K e^{2} \sin ^{2} \beta .
\end{gathered}
$$

Finally, the two solutions $\omega_{1}^{2}$ and $\omega_{2}^{2}\left(\omega_{1}^{2}<\omega_{2}^{2}\right)$ can be obtained by solving (A.15):

$$
\omega_{1,2}^{2}=\frac{c_{3} M+c_{1} J_{y} \mp \sqrt{\left(c_{3} M+c_{1} J_{x}\right)^{2}-4 M J_{x}\left(c_{1} c_{3}-c_{2}^{2}\right)}}{2 M J_{x}} .
$$

Equation (A.17) yields the natural frequencies of the lower and higher rolling vibrations of the stage as

$$
\begin{aligned}
& f_{\mathrm{RL}}=\frac{\omega_{1}}{2 \pi}, \\
& f_{\mathrm{RH}}=\frac{\omega_{2}}{2 \pi} .
\end{aligned}
$$

\section{Conflict of Interests}

The authors declare that there is no conflict of interests regarding the publication of this paper.

\section{References}

[1] M. Ninomiya and S. Kato, "Analysis of linear guide and ball screw stiffness," International Journal of the Japan Society for Precision Engineering, vol. 33, no. 3, pp. 173-177, 1999.

[2] H. Ohta, "Sound of linear guideway type recirculating linear ball bearings," Journal of Tribology, vol. 121, no. 4, pp. 678-685, 1999.

[3] H. Ohta and E. Hayashi, "Vibration of linear guideway type recirculating linear ball bearings," Journal of Sound and Vibration, vol. 235, no. 5, pp. 847-861, 2000.

[4] J. C. Chang, J. S. S. Wu, and J. P. Hung, "Characterization of the dynamic behavior of a linear guideway mechanism," Structural Engineering and Mechanics, vol. 25, no. 1, pp. 1-20, 2007.

[5] Y. Yong-Sub, Y. Y. Kim, J. S. Choi et al., "Dynamic analysis of a linear motion guide having rolling elements for precision positioning devices," Journal of Mechanical Science and Technology, vol. 22 , no. 1 , pp. 50-60, 2008.
[6] Y. L. Lai and J. P. Hung, "The influence of linear guide arrangement on the dynamic characteristics of a gantry type milling machine," Journal of the Chinese Society of Mechanical Engineers, vol. 33, no. 2, pp. 113-122, 2012.

[7] J. Hung, Y. Lai, C. Lin, and T. Lo, "Modeling the machining stability of a vertical milling machine under the influence of the preloaded linear guide," International Journal of Machine Tools and Manufacture, vol. 51, no. 9, pp. 731-739, 2011.

[8] P. Kolar, M. Sulitka, and M. Janota, "Simulation of dynamic properties of a spindle and tool system coupled with a machine tool frame," The International Journal of Advanced Manufacturing Technology, vol. 54, no. 1-4, pp. 11-20, 2011.

[9] G. Bianchi, F. Paolucci, P. van den Braembussche, and H. van Brussel, "Towards virtual engineering in machine tool design," CIRP Annals-Manufacturing Technology, vol. 45, no. 1, pp. 381384, 1996.

[10] Y. Altintas, C. Brecher, M. Week, and S. Witt, "Virtual machine tool," CIRP Annals-Manufacturing Technology, vol. 54, no. 2, pp. 115-138, 2005.

[11] M. Zatarain, E. Lejardi, F. Egaña, and R. Bueno, "Modular synthesis of machine tools," CIRP Annals-Manufacturing Technology, vol. 47, no. 1, pp. 333-x65, 1998.

[12] D. Spath, W. Neithardt, and C. Bangert, "Optimized design with topology and shape optimization," Proceedings of the Institution of Mechanical Engineers B: Journal of Engineering Manufacture, vol. 216, no. 8, pp. 1187-1191, 2002.

[13] G. Reinhart and M. Weissenberger, "Multibody simulation of machine tools as mechatronic systems for optimization of motion dynamics in the design process," in Proceedings of the IEEE/ASME International Conference on Advanced Intelligent Mechatronics (AIM '99), pp. 605-610, Atlanta, Ga, USA, September 1999.

[14] S. Witt, M. Queins, and C. Brecher, "Coupled simulation of structural dynamics and control loop with MSC.ADAMS for the development of high-dynamic machine tools," in Proceedings of the Virtual Product Development Conference, München, Germany, 2004.

[15] M. Law, Y. Altintas, and A. S. Phani, "Rapid evaluation and optimization of machine tools with position-dependent stability," International Journal of Machine Tools and Manufacture, vol. 68, pp. 81-90, 2013.

[16] J. P. Hung, "Load effect on the vibration characteristics of a stage with rolling guides," Journal of Mechanical Science and Technology, vol. 23, no. 1, pp. 89-99, 2009.

[17] THK Technologies Company, "Features of the LM guide," http://www.thk.com/online_cat.

[18] K. J. Johnson, Contact Mechanics, Cambridge University Press, 1985.

[19] D. E. Brewe and B. J. Hamrock, "Simplified solution for elliptical-contact deformation between two elastic solids," Journal of Lubrication Technology, vol. 99, no. 4, pp. 485-487, 1977.

[20] J. A. Greenwood, "Analysis of elliptical Hertzian contacts," Tribology International, vol. 30, no. 3, pp. 235-237, 1997.

[21] Hiwin Technologies Company, "Hiwin ball screw technical information," http://www.ballscrew.tw/support_download_pdf .aspx.

[22] THK Technologies Company, "THK ball screw technical information: ball screw peripheral," http://www.thk.com/online_cat.

[23] J. S.-S. Wu, J.-C. Chang, G.-A. Tsai, C.-Y. Lin, and F.-M. Ou, "The effect of bending loads on the dynamic behaviors of a rolling guide," Journal of Mechanical Science and Technology, vol. 26, no. 3, pp. 671-680, 2012. 


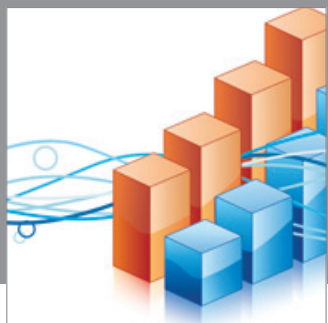

Advances in

Operations Research

mansans

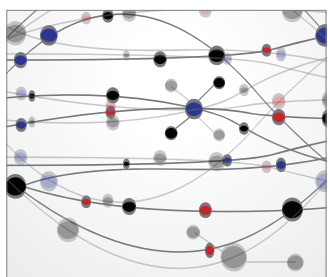

The Scientific World Journal
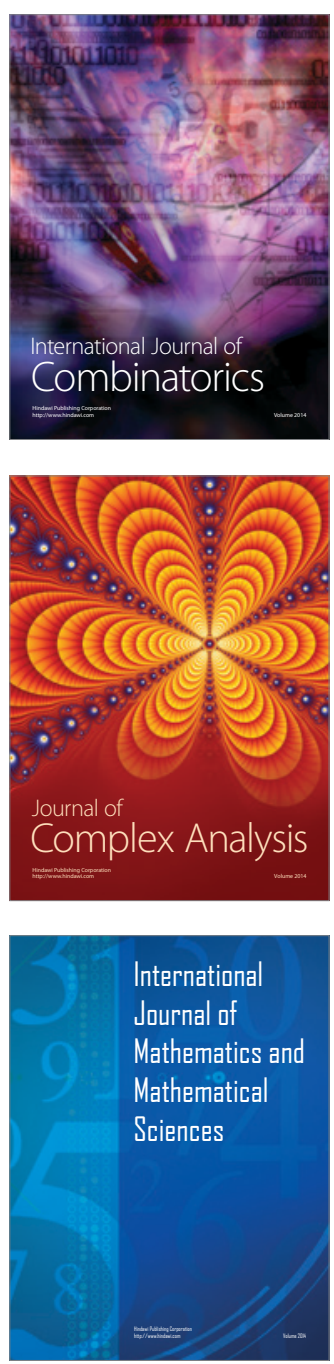
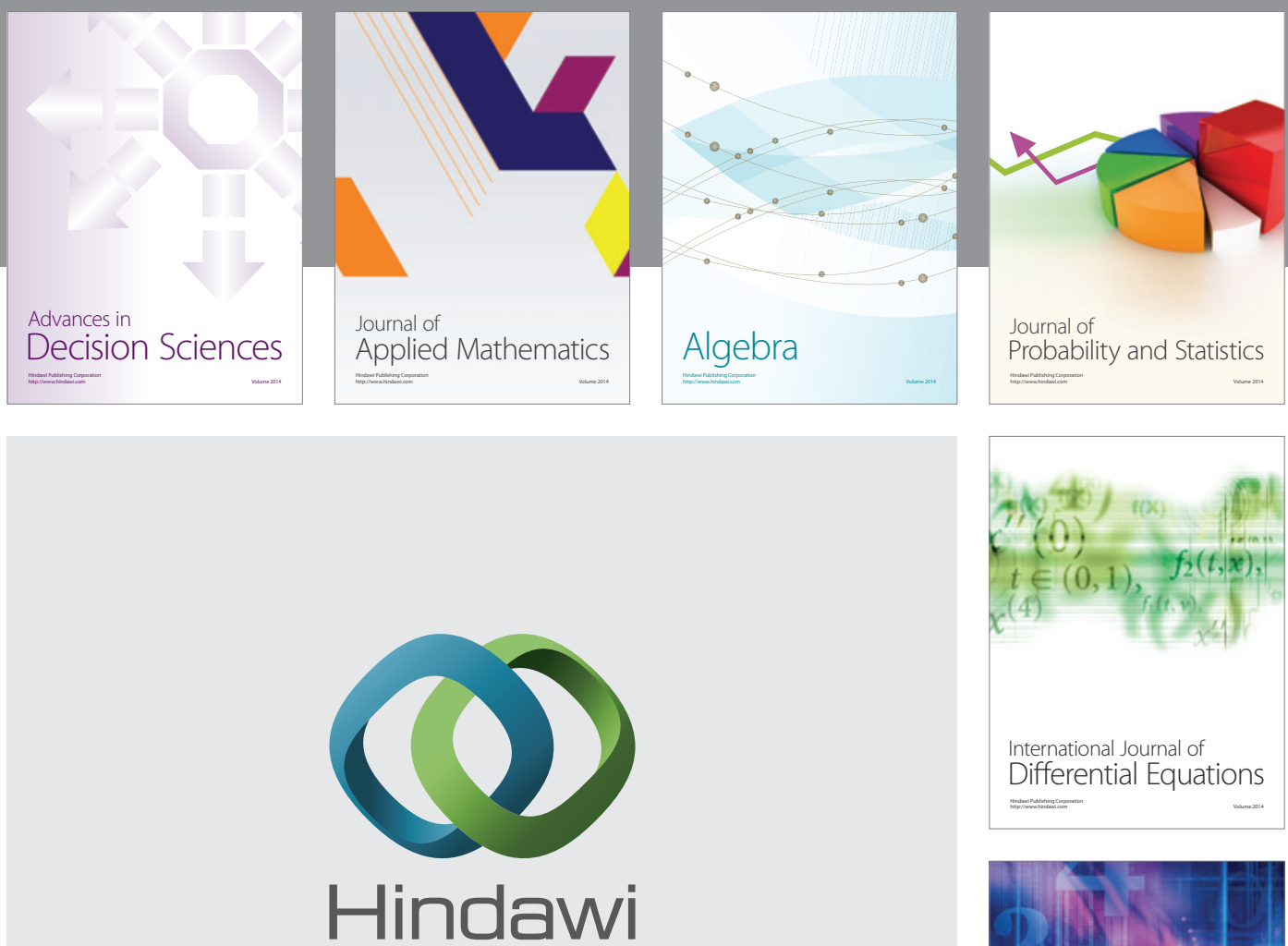

Submit your manuscripts at http://www.hindawi.com
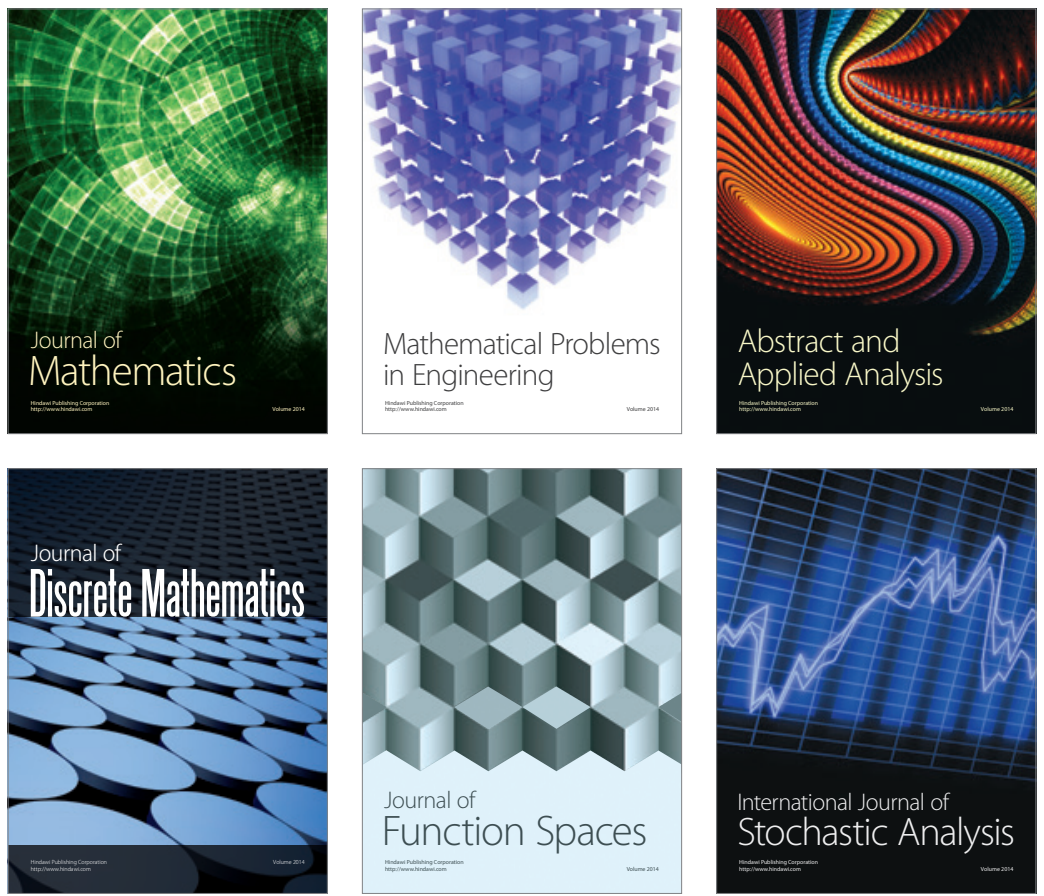

Journal of

Function Spaces

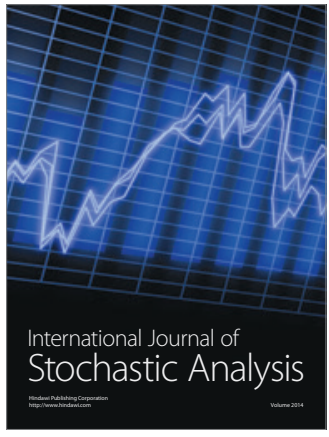

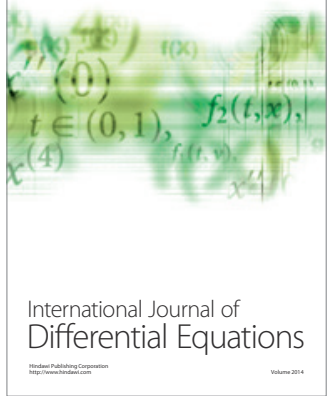
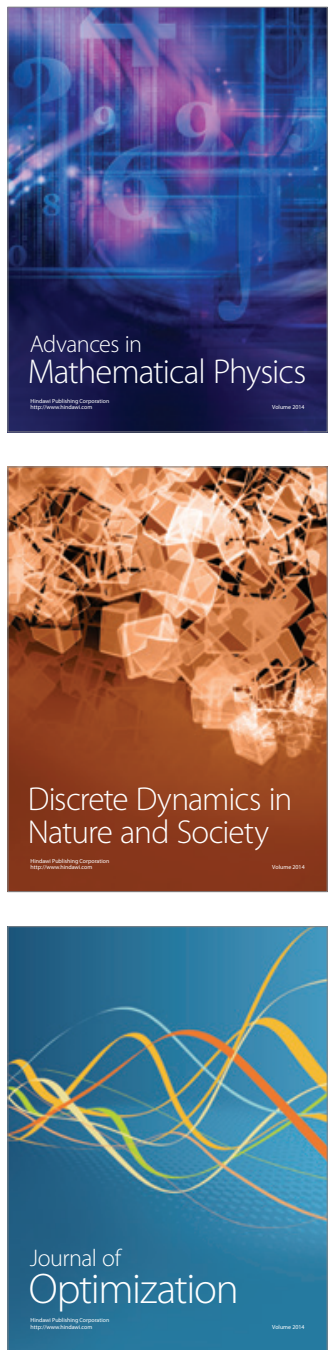